\title{
Morphology control of polysulfone hollow fiber membranes via water vapor induced phase separation
}

\author{
H.A. Tsai ${ }^{\text {a,* }}{ }^{*}$ C.Y. Kuo ${ }^{\text {b }}$, J.H. Lin ${ }^{\text {b }}$, D.M. Wang ${ }^{\text {c }}$, A. Deratani ${ }^{\text {d }}$, \\ C. Pochat-Bohatier ${ }^{\mathrm{e}}$, K.R. Lee ${ }^{\mathrm{f}}$, J.Y. Lai ${ }^{\mathrm{b}}$ \\ ${ }^{a}$ Department of Textile Science, Nanya Institute of Technology, Chung-Li 32034, Taiwan \\ ${ }^{\mathrm{b}} R \& D$ Center for Membrane Technology, Chung Yuan University, Chung-Li, Taiwan \\ ${ }^{\mathrm{c}}$ Department of Chemical Engineering, Taiwan University, Taipei, Taiwan \\ ${ }^{\mathrm{d}}$ Institute Européen des Membranes, Université Montpellier II, Montpellier, France \\ ${ }^{\mathrm{e}}$ Laboratoire de Génie des Procédés, Université Montpellier II, Montpellier, France \\ ${ }^{\mathrm{f}}$ Department of Chemical Engineering, Nanya Institute of Technology, Chung-Li, Taiwan
}

Received 26 May 2005; received in revised form 26 October 2005; accepted 16 November 2005

Available online 20 December 2005

\begin{abstract}
Polysulfone hollow fiber membranes were fabricated by a dry/wet spinning process, with $N$-methyl pyrrolidinone (NMP) and water as solvent and coagulant, respectively. The results indicate that air-gap length and ambient humidity have dramatic effect on the membrane morphology. Macrovoids in the membranes disappeared, reappeared, and redisappeared with increasing air-gap length. Evidence was obtained showing that, because of the high affinity of NMP for water, water vapor was drawn to the dope to induce phase separation, resulting in a nascent structure in the dope before it was immersed in the coagulation bath. We proposed that, shortly after the onset of its phase separation in the air gap, the dope behaved as a transient gel, which can reasonably explain the disappearance and reappearance of macrovoids. On the other hand, when the air gap was long enough to allow phase separation of the entire dope before it reached the coagulation bath, macrovoids redisappeared. In addition, we observed that the air-gap length required for the disappearance and redisappearance of macrovoids decreased with increasing ambient humidity, which can be well reasoned by the decreasing contact time with the humid air needed to bring about phase separation of the dope in the air gap. (c) 2005 Elsevier B.V. All rights reserved.
\end{abstract}

Keywords: Polysulfone; Hollow fiber membrane; Vapor induced phase separation; Macrovoids; Air gap

\section{Introduction}

Membrane processes, with the advantages of easy operation and energy saving, have many applications in separation of mixtures. The applicability of a membrane technique to a separation process strongly depends on if the membrane possessing suitable separation performance can be successfully prepared. Therefore, many researches have concentrated on developing techniques to prepare membranes and to tailor their separation performance. Among the methods for preparation of polymeric membranes, the most widely used one is the phase inversion method [1]. In this method, the phase separation and subsequent solidification (gelation) of the cast polymer solution determine the final mem-

\footnotetext{
* Corresponding author. Tel.: +886 3 4361070x4101.

E-mail address: huian@nanya.edu.tw (H.A. Tsai).
}

brane morphology and the associated separation performance. There are several methods to induce phase separation during membrane forming: change in the solution temperature, the socalled thermally induced phase separation (TIPS), exchange of solvent with nonsolvent (coagulant), the so-called immersion precipitation or nonsolvent induced phase separation (NIPS), and intake of nonsolvent vapor, the so-called vapor induced phase separation (VIPS) [2,3].

With a dry stage for solvent evaporation and a wet stage for exchange of solvent with nonsolvent, the dry/wet phase inversion process produces membranes with various kinds of structure [1]. It is well known that the solvent evaporation in the dry stage affects dramatically the final membrane structure. Less attention has been paid to another phenomenon that can also occur in the dry stage: water vapor in the humid air is drawn to the cast film and thus brings about phase separation (VIPS). In particular, when the polymer solvent used possesses 
low volatility but high affinity for water, in the dry stage the intake of water vapor might prevail over the solvent evaporation. Therefore, to fully understand the forming of polymeric membranes in a dry/wet process, knowledge of VIPS is essential.

Many researches have focused on the VIPS process for preparation of flat-sheet membranes [3-10]. Han and Bhattacharyya [4] prepared polysulfone (PSf) flat-sheet membranes by simply sitting the cast film in humid air, which lead to a liquid-liquid phase separation with nucleation of the polymerpoor phase. Park et al. [3] also adopted the process to fabricate membranes and showed that phase separation only occurred when the relative humidity in air was higher than about $65 \%$. In addition, they observed that the pore size of the resulted membrane was strongly affected by the relative humidity in air. Furthermore, Matsuyama et al. [5,6] showed that, with an increase in relative humidity, they could change the morphology of poly(vinylidenefluoride) (PVDF) membranes from dense to lacy-like, which was believed to be related to the mass increase of the cast film at higher relative humidity. Kang et al. $[7,8]$ also obtained evidence indicating that ambient humidity is an important factor in determining the pore size of membranes. Quantitative analysis of VIPS was conducted as well. Caquineau et al. [9] measured the mass change of cast film during membrane forming and proposed a phenomenological model to explain the morphology evolution caused by VIPS. Khare et al. [10] have developed recently a mathematical model to describe the composition change in the cast film in association with the VIPS process. Another important issue about VIPS is the accompanying phase separation mechanism. Using time-resolved small angle light scattering and phase contrast optical microscopy, Lee et al. [11] were able to show that VIPS actually takes place via spinodal decomposition.

Although the role of VIPS in the forming of flat-sheet membranes was widely studied, as discussed in the preceding paragraph, few articles identified the importance of VIPS in the preparation of hollow fiber membranes. A wet spinning process with an air gap is actually a dry/wet process since both evaporation of solvent and intake of water vapor can occur in the air gap. Therefore, it is reasonable to expect that VIPS occur in the air gap and affect the final morphology of hollow fibers, especially when the polymer solvent has low volatility but high water affinity. However, the role of VIPS in determining the morphology of hollow fibers has yet been investigated. Only some reports [12-15] pointed out that ambient humidity might have influences on membrane morphology. Investigation on the interplay between VIPS and membrane morphology is still needed, to get insight into the morphology control of hollow fibers.

In the present work, we fabricated polysulfone hollow fiber membranes via the dry/wet spinning process, changing the length of air gap and the ambient humidity to demonstrate the effect of VIPS on membrane morphology. Our focus is on how VIPS affects the forming of macrovoids near the outer surface of hollow fibers. A special feature of this work is to lock up the nascent fiber morphology formed in the air gap, by injecting the dope into a bath of liquid nitrogen immediately after the end of the air gap zone. With this method we can compare the morphol- ogy formed in the VIPS stage with the final one, to shed light on the interplay between VIPS and the final morphology of hollow fibers.

\section{Experimental}

\subsection{Materials}

Polysulfone (PSf) (Udel P-3500) was purchased from the AMOCO Performance Products Inc. (Ridgefield, CT, USA). $\mathrm{N}$ methyl pyrrolidinone (NMP) of reagent grade, without further purification, was used as the solvent for PSf, and distilled water was used as its coagulant. In addition, methanol of reagent grade was used to extract the frozen NMP after the dope was injected into liquid nitrogen to luck up the nascent morphology.

\subsection{Spinning of PSf hollow fiber membranes}

PSf chips and NMP were mixed in a flask under agitation to form a homogenous polymer solution with $26 \mathrm{wt} . \%$ of PSf, which was then sit still for at least one day. Next, the homogenous polymer solution was poured into a dope tank and kept there overnight for eliminating the air bubbles formed during the stages of agitation and pouring.

The degassed homogeneous solution was used to fabricate PSf hollow fiber membranes with the dry/wet spinning process. Subject to a pressure of $2 \mathrm{~atm}$, the polymer solution was extruded through a spinneret, with an outside diameter of $0.83 \mathrm{~mm}$ and an inside one of $0.25 \mathrm{~mm}$, to a coagulation bath of water with an adjustable air gap between the spinneret and the water bath. The whole spinning line, from the spinneret to the coagulation bath, was placed in a humidity-and-temperature controlled cabinet (Jwo Ruey Technical Co., Ltd., Taiwan, Model TH-1000), so that the humidity and temperature in the air gap were fixed and could be adjusted during the spinning process. The bore liquid (70/30 NMP/water) was delivered using a syringe pump (500D from ISCO Inc., USA), and there was no external elongation stress except gravity applied to the nascent hollow fiber membranes. After solidification, the fibers were removed from the coagulation bath and put in distilled water for at least three days and then in methanol for $2 \mathrm{~h}$ to remove the residual solvent, which was followed by an air drying stage of about $24 \mathrm{~h}$ at room temperature.

For some experiments, a bath of liquid nitrogen was used instead of the water bath to lock up the nascent morphology that was formed in the air gap due to VIPS. The dope was injected into the liquid nitrogen bath immediately after it passed through the air gap, so that the formed structure in the air gap could be fixed because of fast quenching. In order to examine the lockup morphology by SEM, the NMP contained in the quenched dope had been removed to prevent it from redissolving PSf and destructing the quenched structure when the samples were taken out for drying from the bath of liquid nitrogen. The quenched dope was immersed in methanol at $-90^{\circ} \mathrm{C}$ for $9 \mathrm{~h},-85^{\circ} \mathrm{C}$ for $0.5 \mathrm{~h},-60^{\circ} \mathrm{C}$ for $0.5 \mathrm{~h}$, and $-30^{\circ} \mathrm{C}$ for another $0.5 \mathrm{~h}$ to extract out the frozen NMP. After which, the resulted membrane was air dried for further examination of the structure by SEM. 


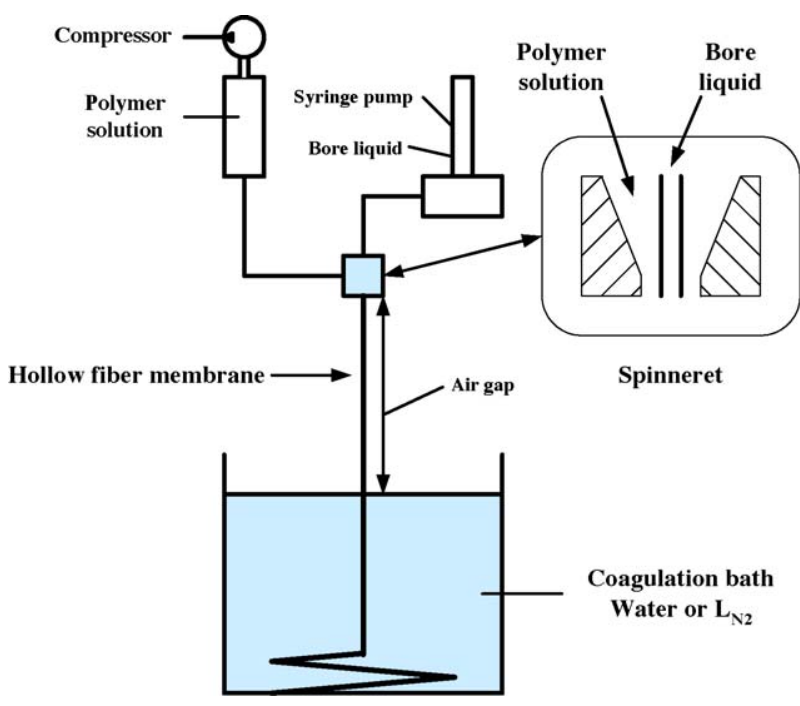

Fig. 1. Schematic representation of the spinning process.

The experimental setup and details of the spinning conditions are shown in Fig. 1 and Table 1, respectively.

\subsection{In situ measurement of the mass variation of a cast film during VIPS}

The degassed $26 \mathrm{wt} . \%$ PSf/NMP solution was cast on a glass plate with a thickness of $300 \mu \mathrm{m}$. The resulted film was then put on an electronic balance that was placed in the cabinet where temperature and humidity were well controlled. The cast film was thus brought into contact with humid air and its mass variation during the contact period was obtained by recording the readings from the balance. The data can be used to estimate the water flux into the cast film during VIPS.

\subsection{Characterization of the morphology of hollow fibers by SEM}

The morphology of the PSf hollow fiber membranes were characterized by a scanning electron microscope (Hitachi, Model: S-3000N and S-4100). Specimens of fiber membranes for SEM were prepared by fracturing the membrane samples in liquid nitrogen. The specimens were covered with a thin layer of Pt by sputter coating before they were sent for SEM analysis.

Table 1

Spinning conditions for preparation of PSf hollow fibers

\begin{tabular}{ll}
\hline Condition & Value \\
\hline Dope solution & PSf/NMP $(26$ wt. $\%)$ \\
Bore liquid (wt. $\%)$ & $\mathrm{NMP} / \mathrm{H}_{2} \mathrm{O}=70 / 30$ \\
External coagulant & $\mathrm{H}_{2} \mathrm{O}$ or liquid nitrogen \\
Dope extrusion pressure & 2 atm $($ volume flow rate $=0.259 \mathrm{ml} / \mathrm{min})$ \\
Bore liquid flow rate $(\mathrm{ml} / \mathrm{min})$ & 0.15 \\
Air gap $(\mathrm{cm})$ & $0-100$ \\
Spinneret diameter $(\mathrm{mm})$ & $\mathrm{OD} / \mathrm{ID}=0.83 / 0.25$ \\
Temperature $\left({ }^{\circ} \mathrm{C}\right)$ & 25 \\
Relative humidity $(\%)$ & $30-90$ \\
\hline
\end{tabular}

\section{Results and discussion}

\subsection{Effect of air-gap length on the morphology of PSf hollow fiber membranes}

The major aim of the present work is to investigate the effect of water vapor in the air gap on the morphology of hollow fiber membranes. It should be noted first that the morphology around the inner surface of fibers is not supposed to be affected by the water vapor in the air gap because it is dominated by the exchange of the polymer solvent with the bore liquid, not like the morphology near the outer surface which can be affected by the water intake from the outer surface of the dope. Therefore, we only concentrated on examining the morphology around the outer surface of hollow fibers, where the effect of water vapor was clearly seen.

Fig. 2(A) shows the SEM of the PSf hollow fiber membrane prepared with no air gap, i.e., the spinneret was in direct contact with the water bath. Obviously, the membrane possessed macrovoids beneath the outer skin. A magnified picture is given in Fig. 2(B) to display more clearly the morphology near the outer surface. Fig. 3 depicts the morphology of the hollow fiber membranes fabricated by varying the length of air gap from 0 to $60 \mathrm{~cm}$, when the relative humidity in the air gap was kept at $30 \%$. The results indicate that the length of air gap affects dramatically the morphology near the outer surface of hollow fibers: macrovoids were clearly seen with no air gap, but totally disappeared with an air gap of $10 \mathrm{~cm}$, reappeared when the air gap increased to $20 \mathrm{~cm}$, became smaller with an air gap longer than $20 \mathrm{~cm}$, and could no longer be seen when the gap was more than $50 \mathrm{~cm}$. Complete suppression of the macrovoids near the outer surface first occurred when the air gap was $10 \mathrm{~cm}$ and took place again when the air gap was longer than $50 \mathrm{~cm}$. From now on, we call the two stages of complete suppression of macrovoids the first suppression and the resuppression, respectively.

A possible reason accounting for the above observation is that the water vapor in the air gap was drawn to the dope. If this is correct, with higher relative humidity in the air gap, we should be able to obtain similar results, but with the disappearance and reappearance of macrovoids occurring at a shorter air gap. Therefore, we adjusted the relative humidity in the air gap to $70 \%$ and $90 \%$ and prepared hollow fiber membranes with different length of air gap. The results are presented below.

\subsection{Effect of the relative humidity in the air gap on the morphology of hollow fiber membranes}

Shown in Fig. 4 are the morphologies of PSf hollow fiber membranes spun with the air gaps from 10 to $60 \mathrm{~cm}$, when the relative humidity in the air gap was kept at $70 \%$. For the case of no air gap $(0 \mathrm{~cm})$, it should be noted that the humidity in the air gap did not affect membrane morphology at all, since the dope had no chance to contact water vapor. Therefore, one can always refer to Fig. 2 for the membrane morphology in the case of no air gap. As expected, the trend shown in Fig. 4 is similar to that in Fig. 3. The macrovoids occurred, disappeared, reappeared, and redisappeared as the air-gap length increased from 0 to $60 \mathrm{~cm}$. 


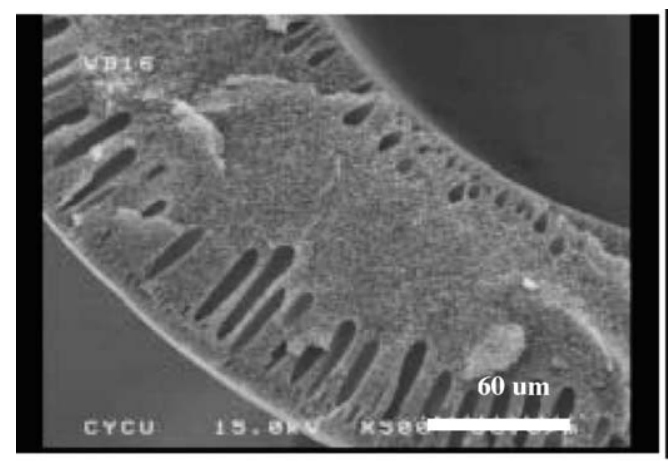

(A)

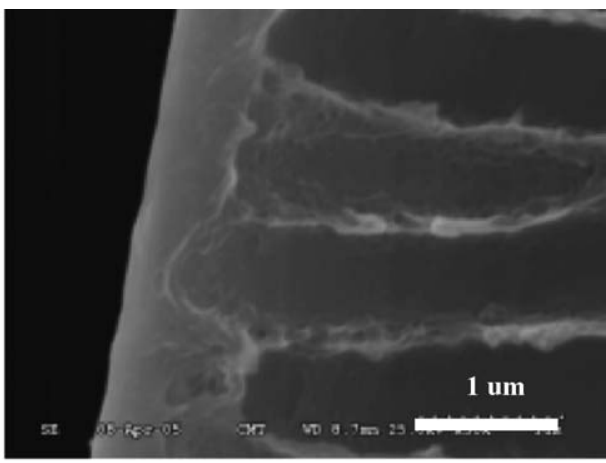

(B)

Fig. 2. Cross-sectional membrane morphology of the PSf hollow fiber prepared with no air gap. Magnification: (A) 500 and (B) $30,000 \times$.

The major difference between Figs. 3 and 4 is the air-gap length required for the resuppression of macrovoids: $40 \mathrm{~cm}$ in the case of $70 \%$ relative humidity but $50 \mathrm{~cm}$ in the case of $30 \%$, indicating that the higher humidity in the air gap shortens the air-gap length required for the resuppression.

The dependence of membrane morphology on air-gap length was also investigated when the relative humidity in the air gap was raised to $90 \%$. The results are presented in Fig. 5. Similarly, with increasing air-gap length, the macrovoids occurred, disappeared, reappeared, and redisappeared. With a relative humidity of $90 \%$, the air-gap length required for the resuppression of macrovoids is shorter compared with the two previous cases:
$30 \mathrm{~cm}$ versus $40 \mathrm{~cm}(70 \%)$ and $50 \mathrm{~cm}(30 \%)$. Moreover, the airgap length required for the first suppression is also shorter: $5 \mathrm{~cm}$ versus $10 \mathrm{~cm}$. The results show that the higher humidity in the air gap shortens the air-gap length required for suppression of macrovoids, for both the first suppression and the resuppression. For clarity the air-gap length needed to completely suppress macrovoids is listed in Table 2.

\subsection{Microstructure of hollow fibers near the outer surface}

To further evaluate the effect of air-gap length and ambient humidity on the morphology of the prepared hollow

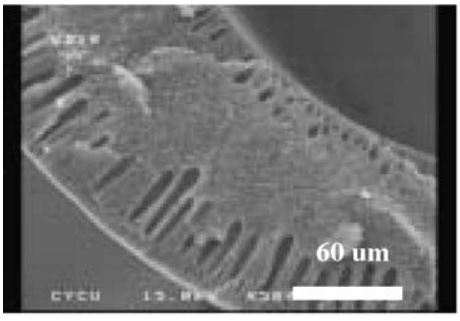

(A)

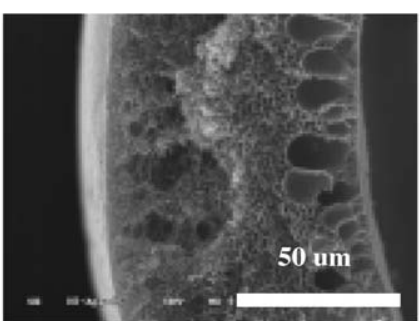

(D)

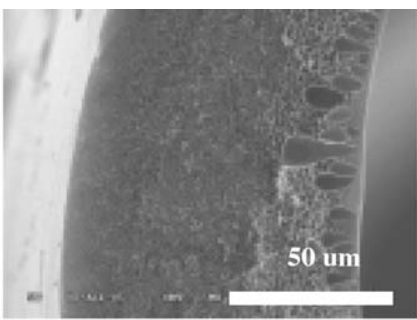

(B)

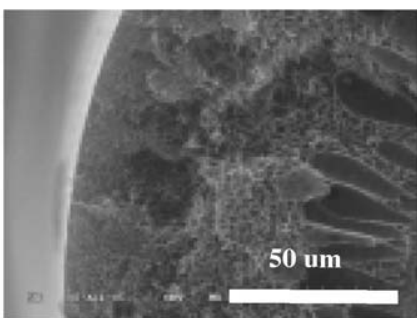

(E)

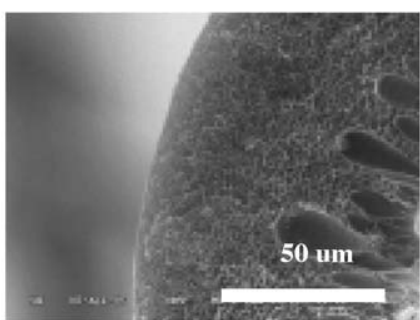

(G)

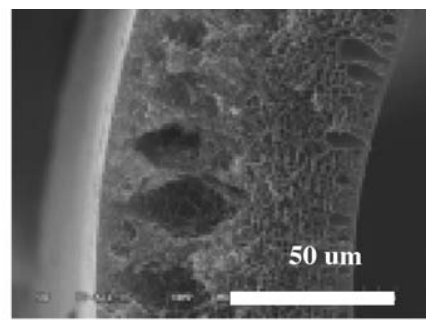

(C)

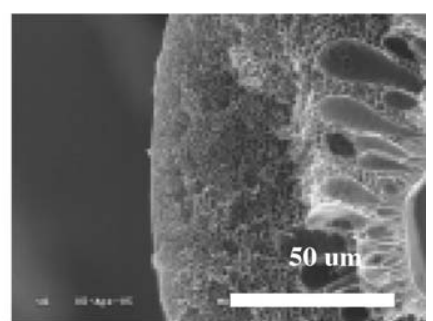

(F)

Fig. 3. Cross-sectional membrane morphology of the PSf hollow fiber prepared with an ambient relative humidity of $30 \%$. Air gap: (A) $0 \mathrm{~cm}$, (B) $10 \mathrm{~cm}$, (C) $20 \mathrm{~cm}$, (D) $30 \mathrm{~cm}$, (E) $40 \mathrm{~cm}$, (F) $50 \mathrm{~cm}$, and (G) $60 \mathrm{~cm}$. 


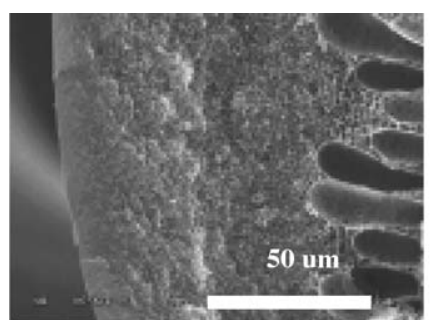

(A)

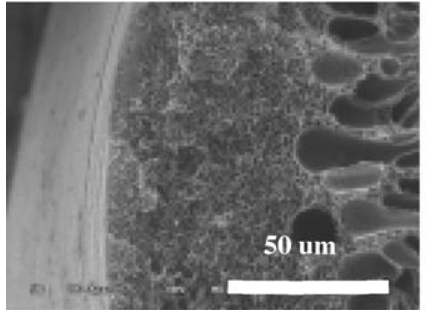

(D)

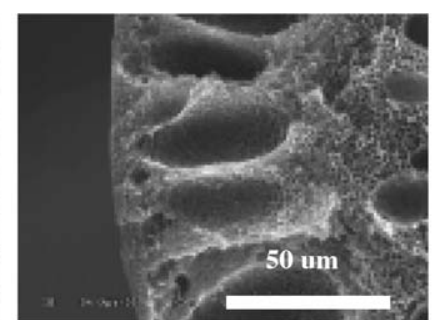

(B)

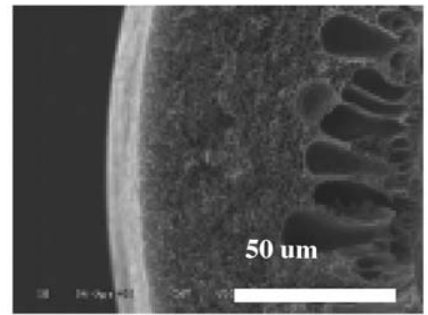

(E)

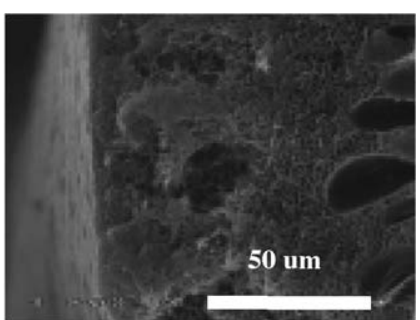

(C)

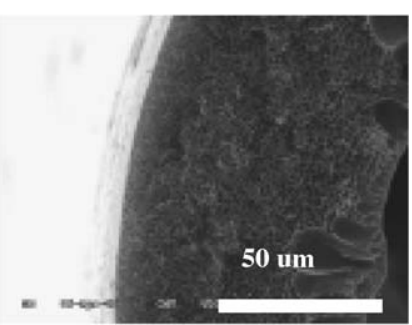

(F)

Fig. 4. Cross-sectional membrane morphology of the PSf hollow fiber prepared with an ambient relative humidity of $70 \%$. Air gap: (A) $10 \mathrm{~cm}$, (B) $20 \mathrm{~cm}$, (C) $30 \mathrm{~cm}$, (D) $40 \mathrm{~cm}$, (E) $50 \mathrm{~cm}$, and (F) $60 \mathrm{~cm}$.

fibers, we examined the microstructure near the outer surface of the fibers. The results are presented in Figs. 6-8. The relative humidity in the air gap was $30 \%$ for the results shown in Fig. 6, 70\% in Fig. 7, and 90\% in Fig. 8. In all the three cases, similar trends were obtained responding to an increase in air-gap length. A dense layer near the outer surface was observed, accompanied with macrovoids, when there was no air gap. The structure became bicontinuous when the air-gap length increased to a value corresponding to the first suppression of macrovoids. Then, the bicontinuous structure turned into the cellular one when macrovoids reappeared.

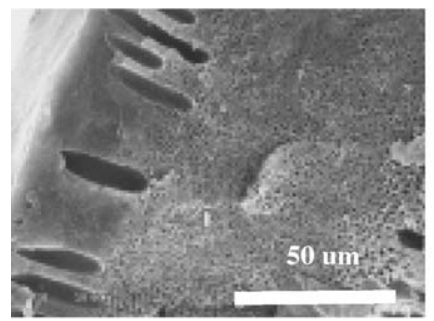

(A)

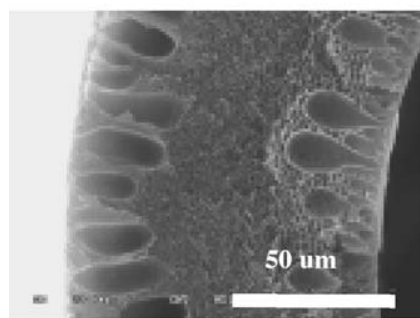

(D)

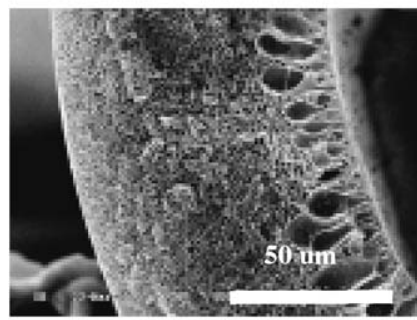

(G)

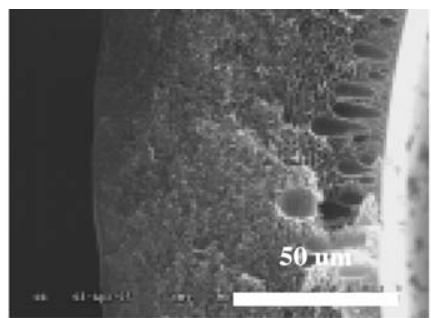

(B)

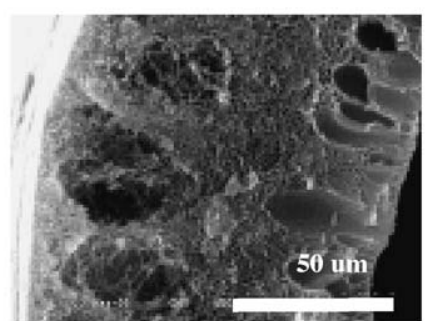

(E)

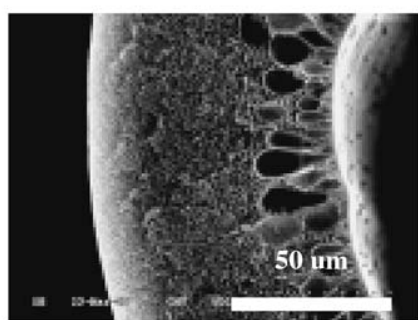

(H)

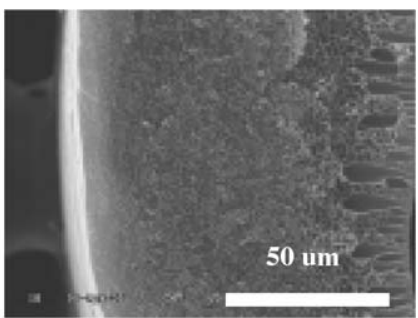

(C)

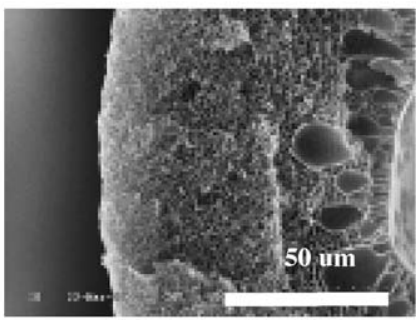

(F)

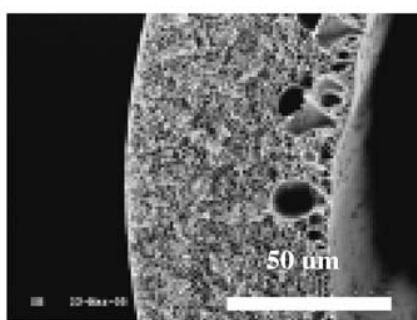

(I)

Fig. 5. Cross-sectional membrane morphology of the PSf hollow fiber prepared with an ambient relative humidity of $90 \%$. Air gap: (A) $2.5 \mathrm{~cm}$, (B) $5 \mathrm{~cm}$, (C) $7.5 \mathrm{~cm}$, (D) $10 \mathrm{~cm}$, (E) $20 \mathrm{~cm}$, (F) $30 \mathrm{~cm}$, (G) $40 \mathrm{~cm}$, (H) $50 \mathrm{~cm}$, and (I) $60 \mathrm{~cm}$. 


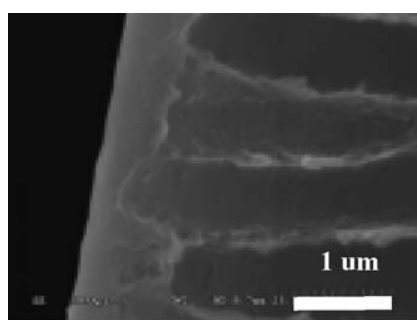

(A)

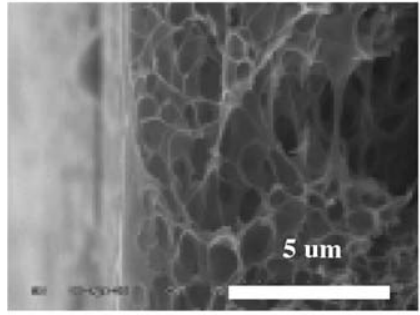

(D)

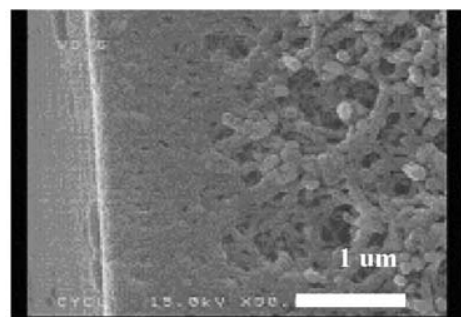

(B)

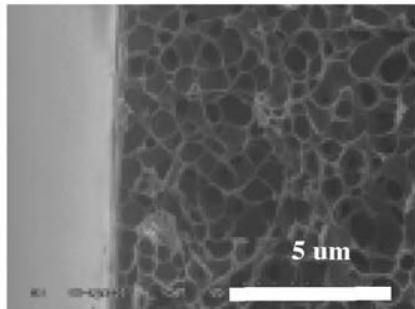

(E)

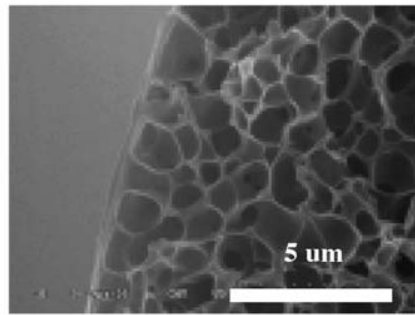

(G)

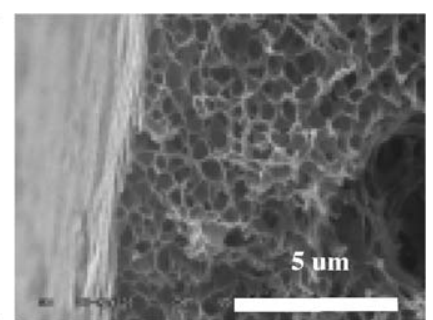

(C)

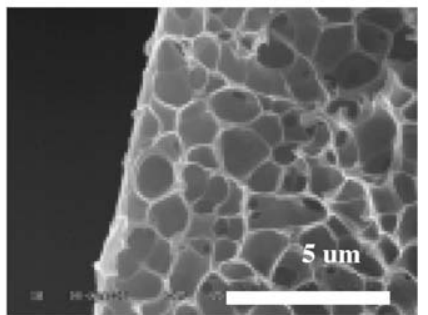

(F)

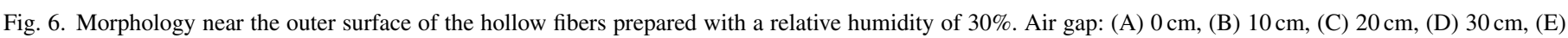
$40 \mathrm{~cm}$, (F) $50 \mathrm{~cm}$, and (G) $60 \mathrm{~cm}$.

The occurrence of a dense layer in association with macrovoids has been widely discussed in the literature [1]. It is believed by many that the fast exchange of solvent with nonsolvent, responsible for the occurrence of macrovoids, also generates a region with high polymer concentration near the interface between solvent and nonsolvent, which then develops into the dense layer. Obviously, the fast exchange of solvent with nonsolvent in the coagulation bath must be disturbed by something happened in the air gap, which will be shown later to be the phase separation induced by the intake of water vapor in the air gap. The relationship between the phase separation in the air gap and the bicontinuous and cellular structures shown in Figs. 6-8 will be discussed in detail in later sections.

\subsection{Water intake in the air gap}

According to the above results, the water vapor in the air gap plays an important role in determining the final morphology of the prepared fibers. In the air gap, solvent evaporation and

Table 2

The air gap length needed to completely suppress macrovoids

\begin{tabular}{lll}
\hline & Suppression $(\mathrm{cm})$ & Resuppression $(\mathrm{cm})$ \\
\hline $30 \%$ RH & 10 & 50 \\
$70 \%$ RH & 10 & 40 \\
$90 \%$ RH & 5 & 30 \\
\hline
\end{tabular}

water intake can both occur and affect the final morphology. However, because of the low volatility of the solvent we used (NMP, boiling point $202{ }^{\circ} \mathrm{C}$ ), solvent evaporation would not be as important as water intake. One method to demonstrate the importance of water intake is to measure the change in the weight of cast film $[9,16]$. Nonetheless, it is very difficult to detect the mass variation of the dope in the air gap. Therefore, we measured the mass variation of a flat-sheet cast film (PSf/NMP) during its contact with humid air, to obtain qualitative understanding about the mass variation in the air gap.

Fig. 9 depicts the mass variation of the flat-sheet cast film when it was in contact with air with different humidity. The results indicate that water intake dominated over solvent evaporation in the initial period, hence resulting in an increase in mass. After some time in the humid air, the film surface was saturated with water; therefore, the mass began to decrease because solvent evaporation now prevailed water intake [16]. In the case of preparation of hollow fibers, because the residence time of the dope in the air gap was very short (about $20 \mathrm{~s}$ with a $60-\mathrm{cm}$ air gap), water intake should dominate over solvent evaporation, as in the initial stage shown in Fig. 9. With the initial slopes of the curves in Fig. 9, the initial fluxes of mass intake were calculated to be $4.4 \times 10^{-7} \mathrm{~g} / \mathrm{cm}^{2} \mathrm{~s}$ for $30 \%$ relative humidity, $2.5 \times 10^{-6} \mathrm{~g} / \mathrm{cm}^{2} \mathrm{~s}$ for $70 \%$, and $4.8 \times 10^{-6} \mathrm{~g} / \mathrm{cm}^{2} \mathrm{~s}$ for $90 \%$. These values are the differences between the fluxes of water intake and solvent evaporation. Since the solvent evaporation rate is small because of its low volatility, the above values are 


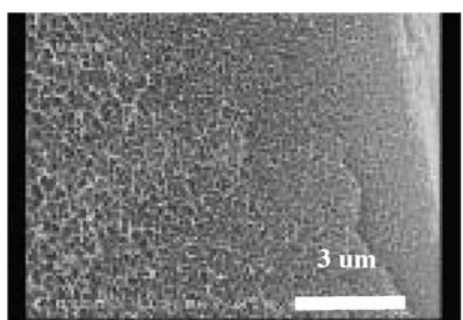

(A)

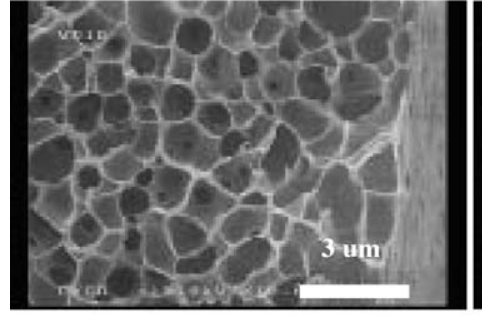

(D)

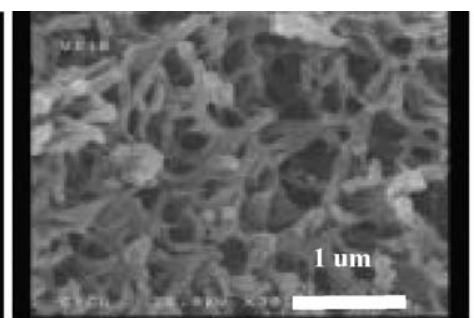

(B)

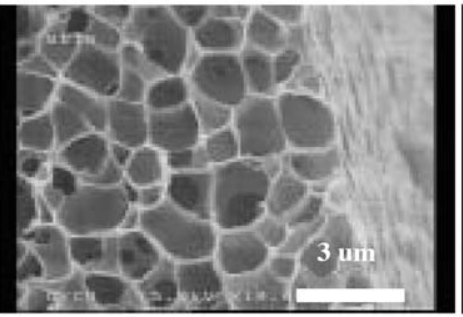

(E)

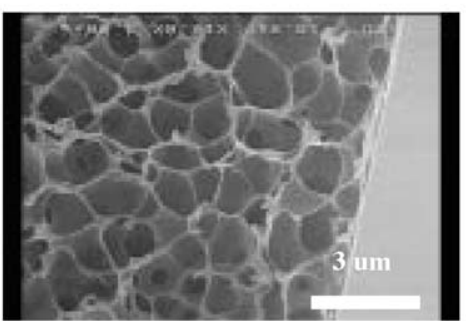

(G)

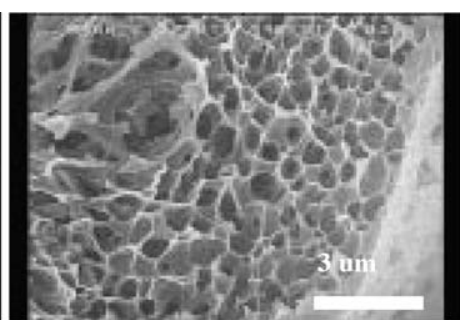

(C)

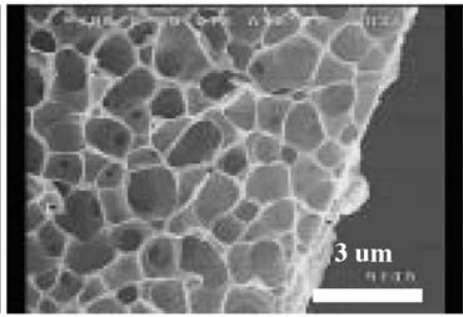

(F)

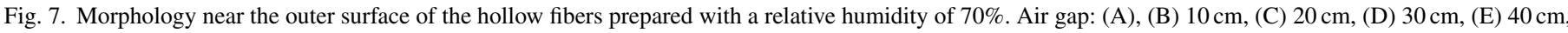
(F) $50 \mathrm{~cm}$, and (G) $60 \mathrm{~cm}$.

reasonable estimates of the initial fluxes of water intake. With the above values, it was found that the amount of water intake was about $5 \times 10^{-5} \mathrm{~g} / \mathrm{cm}^{2}$ after a 20 -s contact with the humid air with a relative humidity of $70 \%$. It was reported [11] that $5 \mathrm{wt} . \%$ of water is enough to bring about the phase separation of PSf/NMP solution. A water intake of $5 \times 10^{-5} \mathrm{~g} / \mathrm{cm}^{2}$ can thus make $1 \times 10^{-3} \mathrm{~g}$ solution phase separate per square centimeter of the film, corresponding to a region with a thickness of about $10 \mu \mathrm{m}$.

It should be noted that the water intake rate in the air gap is supposed to be higher than the above values, which were measured by sitting a cast film in a chamber, because there is a relative velocity between the dope and the air, which can enhance the mass transfer from the air to the dope. On the basis of the above discussion, we believe that, with a $60-\mathrm{cm}$ air gap and a relative humidity of $70 \%$, the water intake from the air gap is enough to generate a phase separation region in the dope with a thickness of several times of $10 \mu \mathrm{m}$.

\subsection{Phase separation in the air gap}

As discussed in the preceding section, the water intake from the surrounding humid air in the air gap was enough to generate phase separation of the dope. Therefore, due to the phase separation, structure might already occur in the dope after it passed through the air gap zone. To examine the structure formed in the air gap, we injected the dope into a liquid nitrogen bath imme- diately after it passed through the air gap, using fast quenching to lock up the nascent structure formed in the air gap. Shown in Fig. 10 are the results obtained when the relative humidity in the air gap was kept at $70 \%$. Obviously, phase separation of the dope did occur in the air gap, evidenced by the structures shown in Fig. 10. In the cases that the air gap was 10 or $20 \mathrm{~cm}$, the structure cannot be clearly seen. But, in the cases that the air gap was longer than $20 \mathrm{~cm}$, the structures generated in the air gap can be clearly recognized.

Fig. 10 provides evidence showing that the dope did contain structure after it passed the air gap and the structure changed with the length of the air gap. Bicontinuous structure was generated with an air gap of $30 \mathrm{~cm}$, which further evolved to cellular one when the air gap was prolonged to $100 \mathrm{~cm}$. Since the bicontinuous structure is a characteristic of spinodal decomposition, our results support that the phase separation mechanism of VIPS is spinodal, as suggested in the work of Lee et al. [11]. The bicontinuous structure developed into the cellular structure if the air gap was long enough for the coarsening process to proceed. Surely, the structure formed in the air gap by VIPS near the outer surface would affect the subsequent mass transfer and structure evolution in the coagulation tank, which we believe is the reason why the air-gap length and ambient humidity can have dramatic effect on the final membrane morphology (Figs. 4 and 7).

Another interesting phenomenon, which is also indicated in Fig. 10, is that the macrovoids near the inner surface actually formed in the air gap. The dope was in contact with the bore 


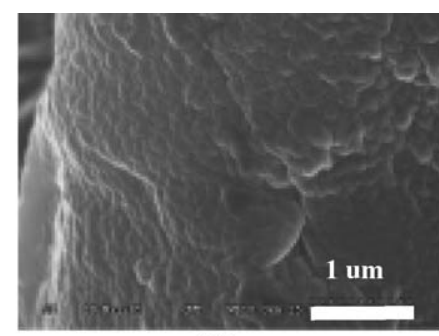

(A)

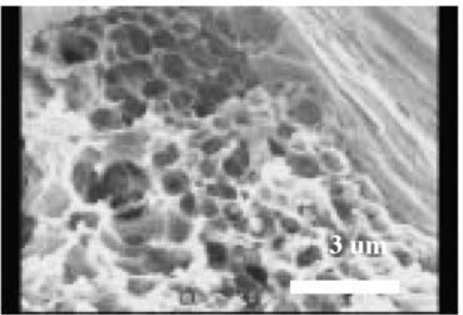

(D)

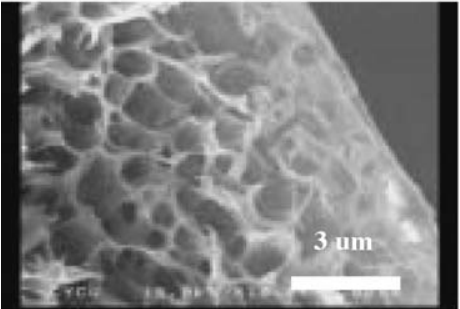

(G)

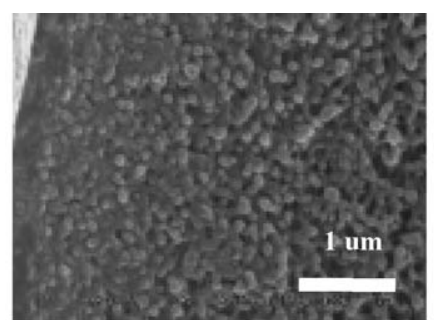

(B)

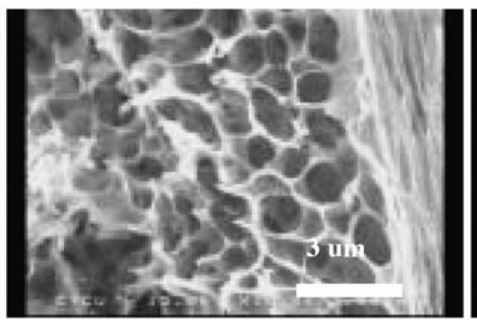

(E)

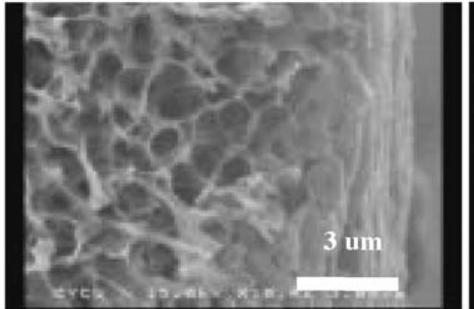

(H)

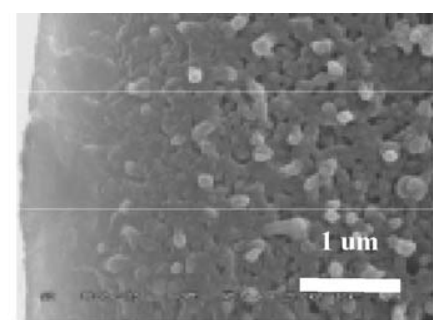

(C)

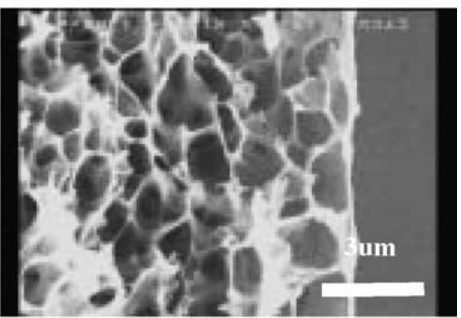

(F)

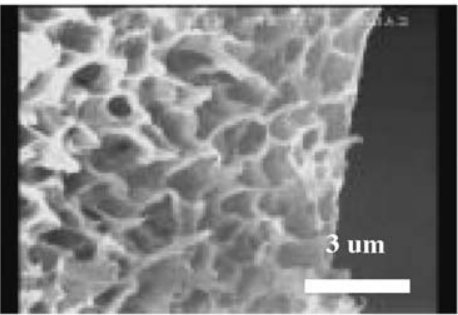

(I)

Fig. 8. Morphology near the outer surface of the hollow fibers prepared with a relative humidity of $90 \%$. Air gap: (A) $2.5 \mathrm{~cm}$, (B) $5 \mathrm{~cm},(\mathrm{C}) 7.5 \mathrm{~cm}$, (D) $10 \mathrm{~cm}$, (E) $20 \mathrm{~cm}$, (F) $30 \mathrm{~cm},(\mathrm{G}) 40 \mathrm{~cm},(\mathrm{H}) 50 \mathrm{~cm}$, and (I) $60 \mathrm{~cm}$.

liquid even in the air gap; therefore, fast exchange of the solvent in the dope with the nonsolvent in the bore liquid can occur and result in macrovoids near the inner surface. On the other hand, in the air gap the outer surface of the dope was in contact with air; fast exchange of solvent and nonsolvent cannot take place to generate macrovoids near the outer surface. Therefore, the marcovoids near the outer surface must form in the coagulation bath, but not in the air gap.

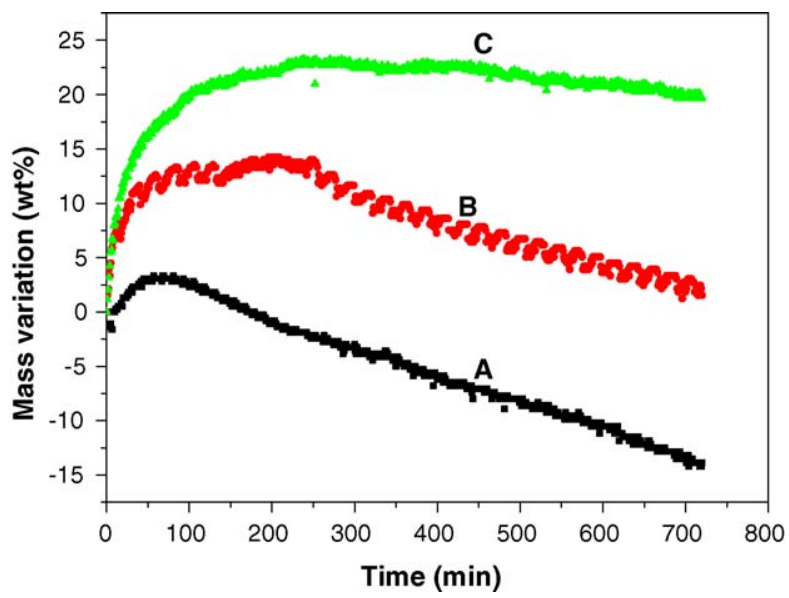

Fig. 9. Mass variation of a flat sheet cast film during its contact with humid air. Polymer solution: 26 wt.\% of PSf in NMP. Relative humidity: (A) 30\%, (B) $70 \%$, and (C) $90 \%$.

\subsection{Suppression of macrovoids}

In the present work, the dope extruded from the spinneret was first in contact with water vapor in the air gap and then immersed in the coagulation bath of water. The preceding section showed that in the air gap VIPS occurred and generated structure in the dope. The structure formed in the air gap would then further develop into its final form in the coagulation bath. By comparing the structure formed in the air gap (Fig. 10) and the final fiber structure (Figs. 4 and 7), we shall be able to get insight into the mechanism of the suppression of macrovoids.

Fig. 4(A) indicates that the macrovoids near the outer surface were completely suppressed with an air gap of $10 \mathrm{~cm}$ when the ambient humidity was kept at $70 \%$. Obviously, the water intake in the $10 \mathrm{~cm}$ air gap has a dramatic effect on the following mass transfer and morphology evolution in the coagulation bath. It was reported that the addition of water in the polymer solution could bring about an increase in the solution viscosity [17]. Thus, one possible reason for the disappearance of macrovoids is an increase in the solution viscosity due to the water intake in the air gap. However, when we raised the solution viscosity by increasing polymer concentration, macrovoids still occurred even when the solution had a viscosity much higher than the one that can be reached with water addition. Therefore, it seems that the increase in viscosity is not a suitable mechanism to account for what we observed. 


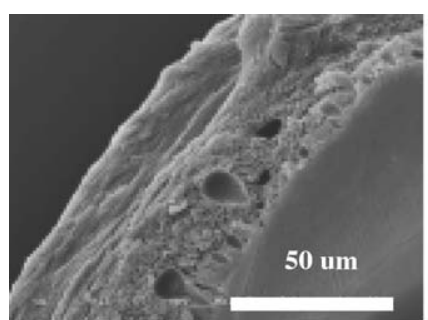

(A)

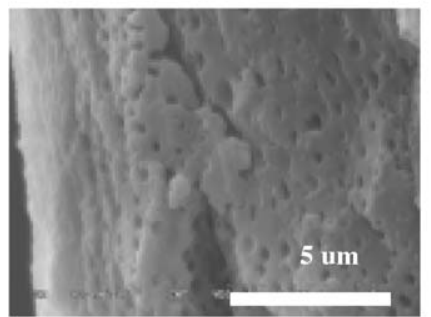

(D)

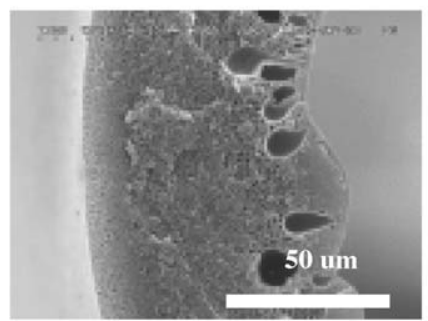

(G)

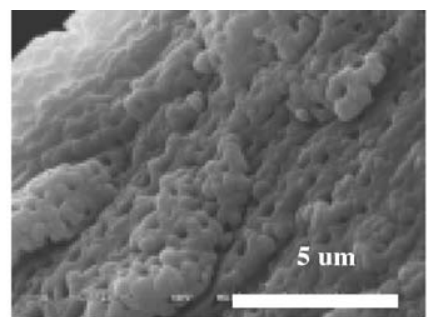

(B)

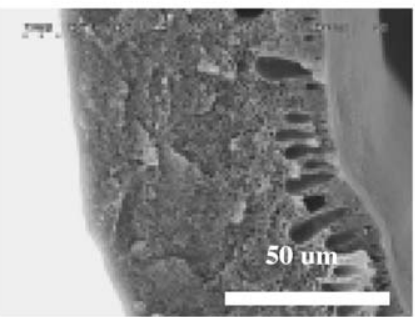

(E)

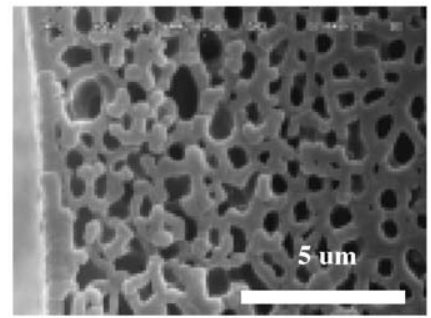

(H)

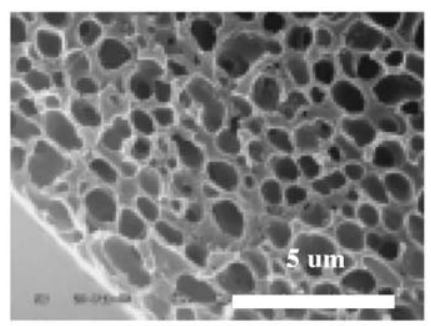

(J)

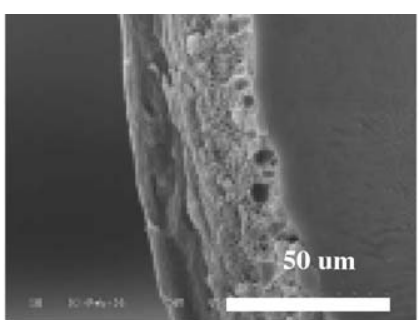

(C)

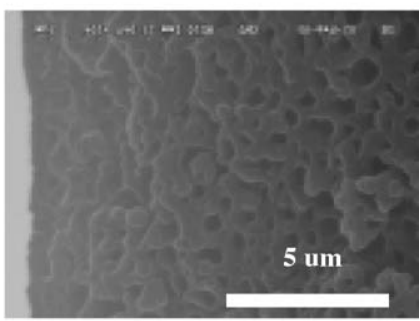

(F)

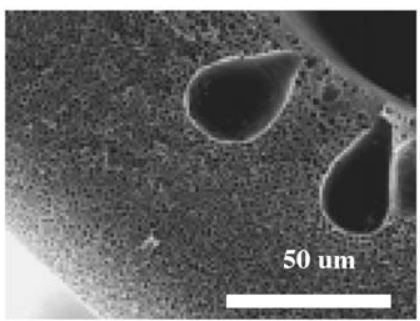

(I)

Fig. 10. The nascent structure formed in the air gap, obtained by quenching the dope in liquid nitrogen. Air gap: (A), (B) $10 \mathrm{~cm}$; (C), (D) $20 \mathrm{~cm}$; (E), (F) $30 \mathrm{~cm}$; (G), (H) $60 \mathrm{~cm}$; (I), (J) $100 \mathrm{~cm}$. Magnification: (A), (C), (E), (G), and (I) 1000×; (B), (D), (F), (H), and (J) 10,000×.

Another possible mechanism is the gelation of the polymerrich phase after VIPS. The formation of a gel layer in the air gap can explain the disappearance of macrovoids because the gel layer can inhibit the fast exchange of solvent and nonsolvent in the coagulation bath. The mechanism can also account for why the bicontinuous structure was always observed accompanying with the first suppression of macrovoids. The gelation of the polymer-rich phase cannot only reduce the exchange rate of solvent and nonsolvent but can also inhibit the subsequent coarsening process of the phase-separated solution. We believe, because of the gelation, the bicontinuous structure formed in the air gap was kept since the subsequent coarsening in the coagulation bath was inhibited. Therefore, the first suppression of macrovoids and the occurrence of bicontinuous structure always took place simultaneously.

One might suspect if phase separation can occur with such a short air gap. According to the calculation based on Fig. 9, a 60-cm air gap can generate a phase separation region with a thickness several times larger than $10 \mu \mathrm{m}$, indicating that the phase separation region corresponding to a 10-cm air gap has a thickness several times larger than $1.6 \mu \mathrm{m}$. In other words, a layer of phase separation region with a thickness of several $\mu \mathrm{m}$ could occur in a $10-\mathrm{cm}$ air gap. Although not very clear, Fig. 10(B) gives evidence to support that phase separation can occur with a $10-\mathrm{cm}$ air gap.

The above mechanism is not complete because it cannot explain why macrovoids reappeared with a longer air gap. When phase separation and gelation can occur in a $10-\mathrm{cm}$ air gap, they should also occur in a 20-cm air gap, implying that macrovoids should also be suppressed with a $20-\mathrm{cm}$ air gap. However, macrovoids reappeared when the air gap was prolonged to $20 \mathrm{~cm}$, as shown in Fig. 4(B). The idea of transient gel would make the above mechanism more reasonable. Tanaka [18] studied the thermally induced phase separation and 
found that, in a short time period after phase separation, the phase-separated solution became a gel because of the elasticity of the polymer-rich phase, resulting in a time period in which coarsening was inhibited. However, after this short period, coarsening was no longer inhibited because the elasticity is important only in a time period shorter than the polymer relaxation time. Therefore, the phase-separated solution was gelled for only a short time period, so he called it "transient gel." The idea of transient gel can well explain why macrovoids were suppressed first but reappeared with an increasing air gap. When phase separation occurred in the air gap due to water intake, a thin gel layer formed which reduced the exchange rate of solvent and nonsolvent in the coagulation bath and thus inhibited the formation of macrovoids. However, because the gel was only transient but not permanent, with a longer air gap, macrovoids reappeared because the phase-separated region was no longer gelled after enough time was given for polymer relaxation. The mechanism of "transient gel" also explains why the reappearance of macrovoids was always associated with larger cells near the outer surface of fibers, compared with the structure formed when the macrovoids were suppressed (first suppression): the subsequent coarsening process was no longer inhibited because the phase-separated region was no longer gelled.

The reappeared macrovoids were getting smaller with an increase in air-gap length. The observation can be well reasoned by an increase in the amount of the intake water form the air gap. After the intake of water form the air gap, a region of phase separation formed from the outer surface down to the center of the dope. In this region, although further coarsening underwent in the subsequent immersion in the coagulation bath when the polymer-rich phase was not gelled (compare Figs. 7 and 10), the nascent structure formed in the air gap was not subject to a dramatic change such as developing into macrovoids, evidenced by the fact that the reappeared macrovoids were only observed at a certain distance beneath the outer surface. The phase separated region grew thicker as the length of air gap increased, thus resulted in smaller macrovoids, which occurred further away from the outer surface. Therefore, macrovoids totally disappeared when the air gap was long enough to allow phase separation to take place, before the dope reached the coagulation bath, in the entire region where the morphology would be affected by the coagulation bath. The above mechanism gives a reasonable explanation of the resuppression of macrovoids.

We proposed in above a mechanism to describe the suppression, reappearance, and resuppression of macrovoids with an increase in air-gap length: the suppression and reappearance were related to the so-called transient gel formed in a short time after phase separation, and the resuppression was resulted from the phase separation of the entire dope before it reached the coagulation bath. Because an increase in relative humidity indicates that less contact time with the humid air is needed to bring about phase separation in the air gap, it is not surprised to see the air-gap length required for the suppression and resuppression of macrovoids decreased with increasing relative humidity, as shown in Figs. 3-5.

\section{Conclusions}

Polysulfone hollow fiber membranes were fabricated by a dry/wet spinning process. The morphology of the fibers was found to be strongly affected by air-gap length and ambient humidity. Macrovoids in the membranes disappeared, reappeared, and redisappeared with increasing air-gap length. The air-gap length required for the suppression and resuppression of macrovoids decreased with increasing relative humidity. Mechanisms were proposed to account for the results observed: the disappearance and reappearance of macrovoids were related to the so-called transient gel formed in a short time after the phase separation induced by the water vapor in the air gap, and the redisappearance was resulted from the phase separation of the entire dope before it reached the coagulation bath.

\section{Acknowledgements}

The authors sincerely thank the Ministry of Economic Affairs and the National Science Council in Taiwan, ROC, for providing financial support for this work.

\section{References}

[1] M.H.V. Mulder, Basic Principles of Membrane Technology, Kluwer Academic Publishers, London, 1996

[2] P. van de Witte, P.J. Dijkstra, J.W.A. van den Berg, B.J. Feijn, Phase separation processes in polymer solutions in relation to membrane formation, J. Membr. Sci. 117 (1996) 1.

[3] H.C. Park, Y.P. Kim, H.Y. Kim, Y.S. Kang, Membrane formation by water vapor induced phase inversion, J. Membr. Sci. 156 (1999) 169.

[4] M.J. Han, D. Bhattacharyya, Changes in morphology and transport characteristics of polysulfone membranes prepared by different demixing conditions, J. Membr. Sci. 89 (1995) 191.

[5] H. Matsuyama, M. Teramoto, R. Nakatani, T. Maki, Membrane formation via phase separation induced by penetration of nonsolvent from vapor phase. I. Phase diagram and mass transfer process, J. Appl. Polym. Sci. 74 (1999) 159

[6] H. Matsuyama, M. Teramoto, R. Nakatani, T. Maki, Membrane formation via phase separation induced by penetration of nonsolvent from vapor phase. II. Membrane morphology, J. Appl. Polym. Sci. 74 (1999) 171.

[7] J.S. Kang, K.Y. Kim, Y.M. Lee, Preparation of microporous chlorinated poly(vinyl chloride) membrane in fabric and the characterization of their pore sizes and pore-size distributions, J. Appl. Polym. Sci. 86 (2002) 1195.

[8] J.S. Kang, K.Y. Kim, Y.M. Lee, Preparation of PVP immobilized microporous chlorinated polyvinyl chloride membranes on fabric and their hydraulic permeation behavior, J. Membr. Sci. 214 (2003) 311.

[9] H. Caquineau, P. Menut, A. Deratani, C. Dupuy, Influence of the relative humidity on film formation by vapor induced phase separation, Polym. Eng. Sci. 43 (2003) 798.

[10] V.P. Khare, A.R. Greenberg, W.B. Krantz, Vapor-induced phase separation-effect of the humid air exposure step on membrane morphology Part I. Insights from mathematical modeling, J. Membr. Sci. 258 (2005) 140.

[11] H.J. Lee, B. Jung, Y.S. Kang, H. Lee, Phase separation of polymer casting solution by nonsolvent vapor, J. Membr. Sci. 245 (2004) 103.

[12] T.S. Chung, X. Hu, Effect of air-gap distance on the morphology and thermal properties of polyethersulfone hollow fibers, J. Appl. Polym. Sci. 66 (1997) 1067. 
[13] T.S. Chung, Z.L. Xu, W.H. Lin, Fundamental understanding of the effect of air-gap distance on the fabrication of hollow fiber membranes, J. Appl. Polym. Sci. 72 (1999) 379.

[14] J.J. Qin, J. Gu, T.S. Chung, Effect of wet and dry-jet wet spinning on the shear-induced orientation during the formation of ultrafiltration hollow fiber membranes, J. Membr. Sci. 182 (2001) 57.

[15] D. Wang, W.K. Teo, K. Li, Preparation and characterization of high-flux polysulfone hollow fibre gas separation membranes, J. Membr. Sci. 204 (2002) 247.
[16] P. Menut, C. Pochat-Bohatier, A. Deratani, C. Dupuy, S. Guilbert, Structure formation of poly (ether-imide) films using nonsolvent vapor induced phase separation: relationship between mass transfer and relative humidity, Desalination 145 (2002) 11.

[17] K.Y. Lin, D.M. Wang, J.Y. Lai, Nonsolvent-induced gelation and its effect on membrane morphology, Macromolecules 35 (2002) 6697.

[18] H. Tanaka, Viscoelastic phase separation, J. Phys.: Condens. Matter. 12 (2000) 207. 